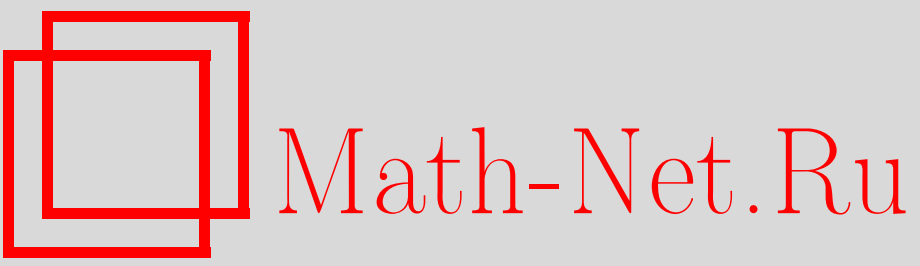

C. Н. Асхабов, Метод максимальных монотонных операторов в теории нелинейных интегро-дифференциальных уравнений типа свертки, Итоги науки и техн. Сер. Соврем. мат. и ее прил. Темат. обз., 2019, том 167, 3-13

DOI: https://doi.org/10.36535/0233-6723-2019-167-3-13

Использование Общероссийского математического портала Math-Net.Ru подразумевает, что вы прочитали и согласны с пользовательским соглашением

http: //www. mathnet.ru/rus/agreement

Параметры загрузки:

IP: 3.80 .181 .102

26 апреля 2023 г., 14:15:14 


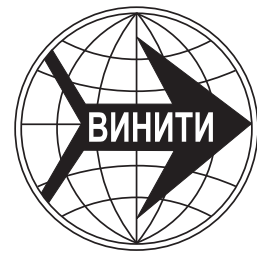

ИТОГИ НАУКИ И ТЕХНИКИ.

Современная математика и ее приложения.

Тематические обзоры.

Том 167 (2019). С. 3-13

DOI: $10.36535 / 0233-6723-2019-167-3-13$

УДК 517.968

\title{
МЕТОД МАКСИМАЛЬНЫХ МОНОТОННЫХ ОПЕРАТОРОВ \\ В ТЕОРИИ НЕЛИНЕЙНЫХ ИНТЕГРО-ДИФФЕРЕНЦИАЛЬНЫХ УРАВНЕНИЙ ТИПА СВЕРТКИ
}

\author{
(c) 2019 г. $\quad$ C. H. ACXАБОВ
}

\begin{abstract}
АннотАция. Методом максимальных монотонных (по Браудеру-Минти) операторов доказаны глобальные теоремы о существовании и единственности решения для различных классов нелинейных интегро-дифференциальных уравнений типа свертки в вещественных пространствах $L_{p}$ при $1<p<\infty$. Приведены следствия, иллюстрирующие полученные результаты.
\end{abstract}

Ключевые слова: положительный оператор, оператор свертки, монотонный оператор, нелинейное интегро-дифференциальное уравнение.

\section{METHOD OF MAXIMAL MONOTONIC OPERATORS IN THE THEORY OF NONLINEAR INTEGRO-DIFFERENTIAL EQUATIONS OF CONVOLUTION TYPE}

\author{
(c) 2019 S. N. ASKHABOV
}

\begin{abstract}
Using the method of maximal monotonic (in the Browder-Minty sense) operators, we prove global theorems on the existence and uniqueness of solutions for various classes of nonlinear integro-differential equations of convolution type in real spaces $L_{p}, 1<p<\infty$, and present illustrative examples.
\end{abstract}

Keywords and phrases: positive operator, convolution operator, monotone operator, nonlinear integro-differential equation.

AMS Subject Classification: 45G10, 47J05

1. Введение. Метод монотонных (по Браудеру-Минти) операторов (см., например, монографию [3] и приведенную в ней библиографию) хорошо известен применительно к нелинейным интегральным уравнениям Гаммерштейна (именно к ним он впервые был применен). Применительно к нелинейным дискретным и интегральным уравнениям типа свертки в пространствах $\ell_{p}$ и $L_{p}$, соответственно, он широко использован в монографии [1]. В данной работе изучаются различные классы нелинейных интегро-дифференциальных уравнений типа свертки в вещественных пространствах Лебега $L_{p}$ на основе более тонкого метода максимальных монотонных операторов (поскольку интегро-дифференциальный оператор свертки определен не на всем пространстве $L_{p}$, то это препятствует применению теоремы Браудера-Минти - основной теоремы теории монотонных операторов; см. [3]). При достаточно широких и легко обозримых ограничениях на нелинейность доказаны глобальные теоремы существования и единственности решения

Работа выполнена при поддержке Российского фонда фундаментальных исследований (проект № 18-41-200001). 
для различных классов нелинейных интегро-дифференциальных уравнений типа свертки. Важно подчеркнуть, что поскольку интегральные уравнения Гаммерштейна имеют ядро общего вида (функция двух переменных), то они изучались в предположении, что линейный интегральный оператор с этим ядром априори является положительным. В случае нелинейных интегральных и интегро-дифференциальных уравнений типа свертки естественно возникает задача нахождения условий на ядро (функция одной переменной), при которых линейные интегральные и интегродифференциальные операторы свертки являются положительными. Решение этой задачи представляет интерес и для гармонического анализа, для которого основополагающую роль играет понятие положительно определенной (по Бохнеру) функции (см. [9]), тесно связанное с понятием положительного оператора. В этой связи в данной работе приведены критерии положительности интегро-дифференциальных операторов свертки, которые используются при доказательстве основных теорем о существовании и единственности решений нелинейных уравнений, порожденных этими операторами. Следует отметить, что теория нелинейных интегро-дифференциальных уравнений типа свертки достаточно хорошо разработана в вольтерровском случае (см. $[10,11])$. Что касается рассматриваемых нами уравнений, то они сравнительно мало изучены, и их исследование имеет не только теоретическое, но и прикладное значение, в частности, для кинетической теории металлов и др. (см., например, статью [8] и приведенную в ней библиографию).

Всюду в работе будем придерживаться принятых в монографии [3] обозначений и определений, касающихся теории монотонных операторов. Пусть $X$-вещественное рефлексивное банахово пространство, $X^{*}$ - сопряженное с ним пространство и оператор $\Lambda$ действует из $X$ в $X^{*}$, т.е. $\Lambda \in\left(X \rightarrow X^{*}\right)$. Обозначим через $\langle y, x\rangle$ значение линейного непрерывного функционала $y \in X^{*}$ на элементе $x \in X$. В частности, если $X$-гильбертово пространство $H$, то $\langle y, x\rangle$ совпадает со скалярным произведением $(y, x)$, где $x, y \in H$. Оператор $\Lambda$ с линейной областью определения $D(\Lambda) \subset X$ называется монотонным, если для любых $u, v \in D(\Lambda)$ выполняется неравенство $\langle\Lambda u-\Lambda v, u-v\rangle \geqslant 0$. Монотонный оператор $\Lambda \in\left(D(\Lambda) \rightarrow X^{*}\right)$ называется максимальным монотонным, если из выполнения неравенства $\langle f-\Lambda v, u-v\rangle \geqslant 0$ для любого $v \in D(\Lambda)$ следует, что $u \in D(\Lambda)$ и $\Lambda u=f$. Если $\Lambda$ - линейный оператор, то определение монотонного оператора совпадает с определением положительного оператора.

Как обычно, через $\mathbb{R}, \mathbb{Z}$ и $\mathbb{N}$ обозначаются соответственно множества всех действительных, целых и натуральных чисел, а через $p^{\prime}=p /(p-1)$ - сопряженное с $p$ число.

\section{2. Критерии положительности интегро-дифференциальных операторов свертки.}

2.1. Интегро-дифференциальный оператор свертки в $L_{p}(-\pi, \pi)$. Рассмотрим в вещественном пространстве Лебега $L_{p}(-\pi, \pi)$, состоящем из $2 \pi$-периодических функций, интегро-дифференциальный оператор свертки

$$
(T u)(x)=\int_{-\pi}^{\pi} h(x-t) \cdot u^{\prime}(t) d t
$$

с областью определения

$$
M_{p}(-\pi, \pi)=\left\{u(x): u(x) \in L_{p}(-\pi, \pi), u^{\prime}(x) \in L_{p^{\prime}}(-\pi, \pi)\right\} .
$$

Всюду далее предполагается, что ядро $h(x) \in L_{1}(-\pi, \pi)$ есть функция, $2 \pi$-периодически продолженная на отрезок $[-2 \pi, 2 \pi]$.

Для выяснения вопроса о том, при каких условиях на ядро $h(x)$ оператор $T$ является положительным в классе $M_{p}(-\pi, \pi)$, приведем необходимые сведения из теории дискретного преобразования Фурье (см. [4]).

Напомним, что дискретным преобразованием Фуръе (изображением) последовательности комплексных чисел $\left\{a_{k}\right\}_{k=-\infty}^{\infty}$ называется соответствующий ей ряд Фурье:

$$
a(x)=\sum_{k=-\infty}^{\infty} a_{k} \cdot \exp (i k x), \quad \text { где } \quad a_{k}=\frac{1}{2 \pi} \int_{-\pi}^{\pi} a(x) \cdot \exp (-i k x) d x, \quad k \in \mathbb{Z} .
$$

Нам понадобятся следующие два равенства (см. [4, с. 233] и [9, с. 158], соответственно): 
(1) формула свертки изображений:

$$
\int_{-\pi}^{\pi} a(x-t) \cdot b(t) d t=2 \pi \sum_{k=-\infty}^{\infty} a_{k} b_{k} \exp (i k x) ;
$$

(2) обобщенное равенство Парсеваля:

$$
\int_{-\pi}^{\pi} a(x) \cdot \overline{b(x)} d x=2 \pi \sum_{k=-\infty}^{\infty} a_{k} \overline{b_{k}}
$$

где

$$
b(x)=\sum_{k=-\infty}^{\infty} b_{k} \exp (i k x), \quad b_{k}=\frac{1}{2 \pi} \int_{-\pi}^{\pi} b(x) \cdot \exp (-i k x) d x,
$$

а черта сверху означает комплексное сопряжение.

Теорема 1. Пусть $1<p<\infty u h(x) \in L_{1}(-\pi, \pi)$. Для того чтобы интегро-дифференииальный оператор свертки $T$ был положительным в классе $M_{p}(-\pi, \pi)$ необходимо и достаточно, чтобы выполнялось условие

$$
\widehat{h}_{s}(k)=\int_{-\pi}^{\pi} h(t) \cdot \sin (k \cdot t) d t \geqslant 0 \quad \text { при всех } k \in \mathbb{N} .
$$

Доказательство. Достаточность. Пусть $u(x) \in M_{p}(-\pi, \pi)$ - произвольная функция и выполнено условие (4). Так как $u^{\prime}(x) \in L_{p^{\prime}}(-\pi, \pi)$ и $h(x) \in L_{1}(-\pi, \pi)$, то из неравенства Юнга для сверток (см. [5, с. 67]) непосредственно вытекает, что $(T u)(x) \in L_{p^{\prime}}(-\pi, \pi)$, т.е. оператор свертки $T$ действует из $M_{p}(-\pi, \pi)$ в $L_{p^{\prime}}(-\pi, \pi)$. Следовательно, функционал $\langle T u, u\rangle$ имеет смысл и принимает конечное значение при любом $u(x) \in M_{p}(-\pi, \pi)$, в силу интегрального неравенства Гельдера.

Докажем положительность оператора $T$. Используя формулу свертки изображений $(2)$, имеем

$$
(T u)(x)=\int_{-\pi}^{\pi} h(x-t) \cdot u^{\prime}(t) d t=2 \pi \sum_{k=-\infty}^{\infty} h_{k} u_{k}^{\prime} \exp (i k x),
$$

где

$$
h_{k}=\frac{1}{2 \pi} \int_{-\pi}^{\pi} h(x) \cdot \exp (-i k x) d x, \quad u_{k}^{\prime}=\frac{1}{2 \pi} \int_{-\pi}^{\pi} u^{\prime}(x) \cdot \exp (-i k x) d x .
$$

Заметим, что

$$
u_{k}^{\prime}=i \cdot k \cdot u_{k} .
$$

В самом деле, так как $u(-\pi)=u(\pi)$ и $\exp (-i k \pi)=\exp (i k \pi)$, применяя формулу интегрирования по частям, получим

$$
u_{k}^{\prime}=\frac{1}{2 \pi}\left[\left.(\exp (-i k x) \cdot u(x))\right|_{-\pi} ^{\pi}-\int_{-\pi}^{\pi} u(x) \cdot \exp (-i k x) \cdot(-i \cdot k) d x\right]=i \cdot k \cdot u_{k} .
$$

Учитывая равенство (6), из (5) имеем

$$
(T u)(x)=2 \pi i \sum_{k=-\infty}^{\infty} k \cdot h_{k} \cdot u_{k} \cdot \exp (i k x),
$$

т.е.

$$
(T u)(x)=\sum_{k=-\infty}^{\infty} T_{k} \cdot \exp (i k x), \quad \text { где } \quad T_{k}=2 \pi i \cdot k \cdot h_{k} \cdot u_{k} .
$$


Используя сначала обобщенное равенство Парсеваля (3), а затем равенство (7), с учетом того, что рассматриваются вещественные функции $u(x)$, получаем

$$
\begin{aligned}
\langle T u, u\rangle=\int_{-\pi}^{\pi}(T u)(x) & \cdot \overline{u(x)} d x=2 \pi \sum_{k=-\infty}^{\infty} 2 \pi i \cdot k \cdot h_{k} \cdot u_{k} \cdot \overline{u_{k}}= \\
& =4 \pi^{2} i \sum_{k=-\infty}^{\infty} k \cdot h_{k} \cdot\left|u_{k}\right|^{2}=4 \pi^{2} i \cdot\left[\sum_{k=-\infty}^{-1} k \cdot h_{k} \cdot\left|u_{k}\right|^{2}+\sum_{k=1}^{\infty} k \cdot h_{k} \cdot\left|u_{k}\right|^{2}\right] .
\end{aligned}
$$

Замечая, что

$$
\begin{gathered}
\left|u_{-k}\right|^{2}=u_{-k} \cdot \overline{u_{-k}}=\left(\frac{1}{2 \pi} \int_{-\pi}^{\pi} u(x) \cdot \exp (i k x) d x\right)\left(\frac{1}{2 \pi} \int_{-\pi}^{\pi} u(x) \cdot \exp (-i k x) d x\right)=\overline{u_{k}} \cdot u_{k}=\left|u_{k}\right|^{2}, \\
h_{k}-h_{-k}=\frac{1}{2 \pi} \int_{-\pi}^{\pi} h(t)(\exp (-i k t)-\exp (i k t)) d t=-\frac{i}{\pi} \int_{-\pi}^{\pi} h(t) \sin (k t) d t=-\frac{i}{\pi} \cdot \widehat{h}_{s}(k),
\end{gathered}
$$

из равенства (8) имеем

$$
\begin{aligned}
\langle T u, u\rangle=4 \pi^{2} i \cdot\left[-\sum_{k=1}^{\infty} k \cdot h_{-k} \cdot\left|u_{-k}\right|^{2}\right. & \left.+\sum_{k=1}^{\infty} k \cdot h_{k} \cdot\left|u_{k}\right|^{2}\right]= \\
& =4 \pi^{2} i \cdot \sum_{k=1}^{\infty} k \cdot\left(h_{k}-h_{-k}\right) \cdot\left|u_{k}\right|^{2}=4 \pi \sum_{k=1}^{\infty} k \cdot \widehat{h}_{s}(k) \cdot\left|u_{k}\right|^{2} .
\end{aligned}
$$

Таким образом,

$$
\langle T u, u\rangle=4 \pi \sum_{k=1}^{\infty} k \cdot \widehat{h}_{s}(k) \cdot\left|u_{k}\right|^{2} \quad \forall u(x) \in M_{p}(-\pi, \pi) .
$$

Из формулы (9) непосредственно вытекает, что оператор $T$ является положительным, если $\widehat{h}_{s}(k) \geqslant 0$, т.е. если выполнено условие $(4)$. Достаточность доказана.

Необходимость. Пусть оператор $T$ является положительным, т.е. $\langle T u, u\rangle \geqslant 0$ при любом $u(x) \in$ $M_{p}(-\pi, \pi)$. Нужно доказать, что тогда выполняется условие (4). Допустим противное, т.е. что существует такое число $k_{0} \in \mathbb{N}$, что $\widehat{h}_{s}\left(k_{0}\right)<0$. Выберем функцию $u(x)=\cos \left(k_{0} x\right)$. Ясно, что $u(x) \in M_{p}(-\pi, \pi)$. Вычислим коэффициенты Фурье этой функции:

$$
u_{k}=\frac{1}{2 \pi} \int_{-\pi}^{\pi} \cos \left(k_{0} x\right) \cdot \cos (k x) d x-i \cdot \frac{1}{2 \pi} \int_{-\pi}^{\pi} \cos \left(k_{0} x\right) \cdot \sin (k x) d x=I_{1}-i \cdot I_{2} .
$$

Легко видеть, что

$$
\begin{aligned}
& I_{1}=\frac{1}{4 \pi} \int_{-\pi}^{\pi} \cos \left(\left(k+k_{0}\right) x\right) d x+\frac{1}{4 \pi} \int_{-\pi}^{\pi} \cos \left(\left(k-k_{0}\right) x\right) d x= \begin{cases}1 / 2, & \text { если } k= \pm k_{0}, \\
0, & \text { если } k \neq \pm k_{0},\end{cases} \\
& I_{2}=\frac{1}{4 \pi} \int_{-\pi}^{\pi} \sin \left(\left(k+k_{0}\right) x\right) d x+\frac{1}{4 \pi} \int_{-\pi}^{\pi} \sin \left(\left(k-k_{0}\right) x\right) d x=0 \quad \forall k \in \mathbb{N} .
\end{aligned}
$$

Подставив в (10), получим такую последовательность $\left\{u_{k}\right\}_{k=-\infty}^{\infty}$, что

$$
u_{-k_{0}}=u_{k_{0}}=\frac{1}{2} \quad \text { и } \quad u_{k}=0 \quad x \text { при всех } \quad k \neq \pm k_{0}
$$

(непосредственно легко проверяется, что изображением этой последовательности действительно является функция $\left.u(x)=\cos \left(k_{0} x\right)\right)$. 
Наконец, по формуле (9), учитывая равенства (11), получаем

$$
\langle T u, u\rangle=4 \pi k_{0} \cdot \widehat{h}_{s}\left(k_{0}\right) \cdot \frac{1}{4}=\pi k_{0} \cdot \widehat{h}_{s}\left(k_{0}\right)<0,
$$

что противоречит положительности оператора $T$. Необходимость, а вместе с ней и теорема, доказаны.

Примером функции, удовлетворяющей условию (4), может служить монотонно невозрастающая функция. Именно, если в качестве основного интервала взять $(0,2 \pi)$, то нетрудно показать (см. $[7$, с. 46$])$, что $\widehat{h}_{s}(k) \geqslant 0$, если функция $h(x)$ не возрастает в интервале $(0,2 \pi)$.

2.2. Интегро-дифференциальный оператор свертки в $L_{p}(\mathbb{R})$. Следующая теорема является аналогом теоремы 1 и относится к случаю вещественных пространств $L_{p}(\mathbb{R}), 1 \leqslant p<\infty$, с обычной нормой

$$
\|u\|_{p}=\left(\int_{-\infty}^{\infty}|u(x)|^{p} d x\right)^{1 / p}
$$

В отличие от п. 2.1, где использовалось дискретное преобразование Фурье, доказательство этой теоремы существенно будет опираться на теорию интегрального преобразования Фурье.

Теорема 2. Пусть $1<p<\infty$ и ядро $h(x) \in L_{1}(\mathbb{R})$. Для того, чтобы интегро-дифберенииальный оператор свертки

$$
(\mathbb{T} u)(x)=\int_{-\infty}^{\infty} h(x-t) \cdot u^{\prime}(t) d t
$$

был положительным в классе $M_{p}(\mathbb{R})=\left\{u(x): u(x) \in L_{p}(\mathbb{R}), u^{\prime}(x) \in L_{p^{\prime}}(\mathbb{R})\right\}$, необходимо $и$ достаточно, чтобы выполнялось условие

$$
\widehat{h}_{s}(x)=\frac{1}{\sqrt{2 \pi}} \int_{-\infty}^{\infty} h(t) \cdot \sin (x \cdot t) d t \geqslant 0 \quad \text { при всех } \quad x \in[0, \infty) .
$$

Доказательство. Достаточность. Так как $h(x) \in L_{1}(\mathbb{R}), u^{\prime}(x) \in L_{p^{\prime}}(\mathbb{R})$, то, в силу неравенства Юнга (см. $[1$, с. 30$]),(\mathbb{T} u)(x) \in L_{p^{\prime}}(\mathbb{R})$ при любом $p^{\prime} \in(1, \infty)$, т.е. оператор $\mathbb{T}$ действует из $M_{p}(\mathbb{R})$ в $L_{p^{\prime}}(\mathbb{R})$. Следовательно, функционал $\langle\mathbb{T} u, u\rangle$ имеет смысл и принимает конечное значение при любом $u(x) \in M_{p}(\mathbb{R})$ в силу интегрального неравенства Гельдера.

Докажем положительность оператора $\mathbb{T}$. Обозначим через $\widehat{u}(x)$ преобразование Фурье функции $u(x) \in L_{2}(\mathbb{R})($ см. $[6$, с. 55$]):$

$$
\widehat{u}(x)=\underset{N \rightarrow \infty}{\lim } \frac{1}{\sqrt{2 \pi}} \int_{-N}^{N} u(t) \cdot \exp (-i x t) d t
$$

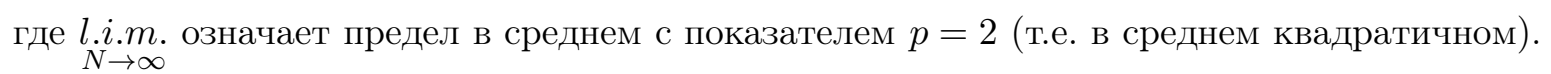

Известно $($ см. $[6])$, что $\widehat{u}(x) \in L_{2}(\mathbb{R})$, если $u(x) \in L_{2}(\mathbb{R})$, и для любых $u(x), v(x) \in L_{2}(\mathbb{R})$ справедливо обобщенное равенство Парсеваля $(u, v)=(\widehat{u}, \widehat{v})$, т.е.

$$
\int_{-\infty}^{\infty} u(x) \cdot \overline{v(x)} d x=\int_{-\infty}^{\infty} \widehat{u}(x) \cdot \overline{\widehat{v}(x)} d x
$$

где черта сверху означает комплексное сопряжение. Кроме того, если $h(x) \in L_{1}(\mathbb{R}), u(x) \in L_{2}(\mathbb{R})$, то для преобразования Фурье свертки $h * u$ справедливо равенство

$$
\widehat{(h * u)}(x)=\sqrt{2 \pi} \widehat{h}(x) \cdot \widehat{u}(x),
$$


где

$$
\widehat{h}(x)=\frac{1}{\sqrt{2 \pi}} \int_{-\infty}^{\infty} h(t) \cdot \exp (-i x t) d t
$$

Легко показать, что $\lim _{|x| \rightarrow \infty} u(x)=0$, если $u(x) \in M_{p}(\mathbb{R})$. В самом деле, так как

$$
|u(x+\Delta x)-u(x)|=\left|\int_{x}^{x+\Delta x} u^{\prime}(t) d t\right| \leqslant(|\Delta x|)^{1 / p}\left(\int_{-\infty}^{\infty}\left|u^{\prime}(t)\right|^{p^{\prime}}\right)^{1 / p^{\prime}} \rightarrow 0 \quad x \text { при } \quad \Delta x \rightarrow 0,
$$

то функция $u(x)$ непрерывна, т.е. $u(x) \in C(\mathbb{R})$. Докажем, что $\lim _{x \rightarrow \infty} u(x)=0$. Так как $u(x) \in C(\mathbb{R})$ и

$$
u^{2}(x)-u^{2}(0)=2 \int_{0}^{x} u(t) \cdot u^{\prime}(t) d t \leqslant 2 \int_{-\infty}^{\infty}\left|u(t) \cdot u^{\prime}(t)\right| d t \leqslant 2\|u\|_{p} \cdot\left\|u^{\prime}\right\|_{p^{\prime}}
$$

то существует конечный предел $\lim _{x \rightarrow \infty}|u(x)|=A$. Осталось показать, что $A=0$. Допустим противное, что $A>0$. Тогда найдется такое достаточно большое число $c>0$, что при всех $x>c$ будет выполняться неравенство $|u(x)|>A / 2$ и, значит,

$$
\|u\|_{p}^{p} \geqslant \int_{-\infty}^{\infty}\left(\frac{A}{2}\right)^{p} d x=\infty
$$

что невозможно, так как $u(x) \in L_{p}(\mathbb{R})$. Значит, $A=0$ и, следовательно, $\lim _{x \rightarrow \infty} u(x)=0$.

Аналогично доказывается, что и $\lim _{x \rightarrow-\infty} u(x)=0$, если $u(x) \in M_{p}(\mathbb{R})$.

Пусть $u(x) \in C_{0}^{\infty}(\mathbb{R})$, т.е. является финитной бесконечно дифференцируемой на всей действительной оси $\mathbb{R}$ функцией. Тогда (ср. [6, с. 57]), интегрируя по частям, получим

$$
\widehat{u^{\prime}}(x)=i \cdot x \cdot \widehat{u}(x) .
$$

Используя равенства $(13)-(15)$, с учетом того, что $u(x) \in C_{0}^{\infty}(\mathbb{R})$ - вещественная функция, имеем

$$
\begin{aligned}
(\mathbb{T} u, u)=\int_{-\infty}^{\infty}\left(h * u^{\prime}\right)(x) \cdot \overline{u(x)} d x= & \int_{-\infty}^{\infty}\left(\widehat{h * u^{\prime}}\right)(x) \cdot \overline{\widehat{u}(x)} d x= \\
=\sqrt{2 \pi} \int_{-\infty}^{\infty} \widehat{h}(x) \widehat{u^{\prime}}(x) \overline{\widehat{u}(x)} d x= & \sqrt{2 \pi} \int_{-\infty}^{\infty} \widehat{h}(x) i x \widehat{u}(x) \overline{\widehat{u}(x)} d x=i \sqrt{2 \pi} \int_{-\infty}^{\infty} \widehat{h}(x) x|\widehat{u}(x)|^{2} d x= \\
& =i \sqrt{2 \pi}\left[\int_{-\infty}^{\infty} \widehat{h_{c}}(x) x|\widehat{u}(x)|^{2} d x-i \int_{-\infty}^{\infty} \widehat{h_{s}}(x) x|\widehat{u}(x)|^{2} d x\right],
\end{aligned}
$$

где косинус-преобразование Фурье

$$
\widehat{h_{c}}(x)=\frac{1}{\sqrt{2 \pi}} \int_{-\infty}^{\infty} h(t) \cdot \cos (x t) d t
$$

есть четная функция, а синус-преобразование Фурье

$$
\widehat{h_{s}}(x)=\frac{1}{\sqrt{2 \pi}} \int_{-\infty}^{\infty} h(t) \cdot \sin (x t) d t
$$


- функция нечетная на действительной оси $\mathbb{R}$. Замечая, что

$$
\begin{aligned}
|\widehat{u}(-x)|^{2}=\widehat{u}(-x) \cdot \overline{\widehat{u}(-x)}=\left(\frac{1}{\sqrt{2 \pi}} \int_{-\infty}^{\infty} u(t) \cdot \exp (i x t) d t\right)\left(\frac{1}{\sqrt{2 \pi}} \int_{-\infty}^{\infty} u(t) \cdot \exp (-i x t) d t\right) & = \\
& =\overline{\widehat{u}(x)} \cdot \widehat{u}(x)=|\widehat{u}(x)|^{2}
\end{aligned}
$$

и, значит, $x \cdot|\widehat{u}(x)|^{2}$ - нечетная на оси $\mathbb{R}$ функция, из равенства (16) получаем

$$
(\mathbb{T} u, u)=\sqrt{2 \pi} \int_{-\infty}^{\infty} \widehat{h_{s}}(x) x|\widehat{u}(x)|^{2} d x
$$

или, что то же самое,

$$
(\mathbb{T} u, u)=2 \sqrt{2 \pi} \int_{0}^{\infty} \widehat{h_{s}}(x) x|\widehat{u}(x)|^{2} d x .
$$

Из равенства (18) следует, что $(T u, u) \geqslant 0$ для любого $u(x) \in C_{0}^{\infty}(\mathbb{R})$, если $\widehat{h_{s}}(x) \geqslant 0$ для почти всех $x \in[0, \infty)$. Так как по теореме Римана-Лебега (см. [6, с. 42]) $\widehat{h_{s}}(x)$ есть непрерывная на всей оси $\mathbb{R}$ функция, то условие, что $\widehat{h_{s}}(x) \geqslant 0$ для почти всех $x \in[0, \infty)$, равносильно условию, что $\widehat{h_{s}}(x) \geqslant 0$ для всех $x \in[0, \infty)$.

Таким образом, если $h(x) \in L_{1}(\mathbb{R})$ и $\widehat{h_{s}}(x) \geqslant 0$ для всех $x \in[0, \infty)$, то справедливо неравенство $(\mathbb{T} u, u) \geqslant 0$ для любого $u(x) \in C_{0}^{\infty}(\mathbb{R})$. Поскольку множество $C_{0}^{\infty}(\mathbb{R})$ всюду плотно в классе $M_{p}(\mathbb{R})$ и $\langle\mathbb{T} u, u\rangle$ - линейный непрерывный функционал, то неравенство $(\mathbb{T} u, u) \geqslant 0$ будет выполняться и всюду в классе $M_{p}(\mathbb{R})$.

Итак мы доказали, что если выполнено условие

$$
h(x) \in L_{1}(\mathbb{R}) \quad \text { и } \quad \widehat{h_{s}}(x)=\frac{1}{\sqrt{2 \pi}} \int_{-\infty}^{\infty} h(t) \cdot \sin (x t) d t \geqslant 0 \quad \forall x \in[0, \infty),
$$

то интегро-дифференциальный оператор свертки $\mathbb{T}$ является положительным, т.е. выполняется неравенство

$$
\langle\mathbb{T} u, u\rangle \geqslant 0 \quad \forall u(x) \in M_{p}(\mathbb{R}) .
$$

Необходимость. Докажем, наконец, что условие (12) также и необходимо для положительности оператора $\mathbb{T}$. Пусть неравенство $(20)$ выполнено. Нужно доказать, что тогда $\widehat{h_{s}}(x) \geqslant 0$ для всех $x \in[0, \infty)$, т.е. выполнено условие (12). Допустим противное, т.е. что условие (12) не выполняется, т.е. существует такая точка $x_{0} \in[0, \infty)$, что $\widehat{h}_{s}\left(x_{0}\right)<0$. Ясно, что $x_{0}>0$, так как очевидно, что $\widehat{h_{s}}(0)=0$. Поскольку по теореме Римана-Лебега $\widehat{h_{s}}(x)$ есть непрерывная на всей оси $\mathbb{R}$ функция, то найдется такая $\varepsilon$-окрестность $U_{\varepsilon}\left(x_{0}\right)=\left\{x: x>0,\left|x-x_{0}\right|<\varepsilon\right\}, \varepsilon>0$, точки $x_{0}$, что будет выполняться неравенство

$$
\widehat{h_{s}}(x)<0 \quad \forall x \in U_{\varepsilon}\left(x_{0}\right) .
$$

Выберем такую функцию $u(x) \in M_{p}(\mathbb{R})$, что $\widehat{u}(x)=0$, если $x \notin U_{\varepsilon}\left(x_{0}\right)$ и $\widehat{u}(x) \neq 0$, если $x \in U_{\varepsilon}\left(x_{0}\right)$. Тогда, учитывая, что для всех $x \in U_{\varepsilon}\left(x_{0}\right)$ выполняются строгие неравенства $\widehat{h_{s}}(x)<0, x>0$, и $|\widehat{u}(x)|>0$, по формуле (5) получим

$$
\langle\mathbb{T} u, u\rangle=\sqrt{2 \pi} \int_{-\infty}^{\infty} \widehat{h_{s}}(x) x|\widehat{u}(x)|^{2} d x=\sqrt{2 \pi} \int_{U_{\varepsilon}\left(x_{0}\right)} \widehat{h_{s}}(x) x|\widehat{u}(x)|^{2} d x<0,
$$

что противоречит неравенству (20). Необходимость, а вместе с ней и теорема, доказана.

Примером ядра $h(x)$, удовлетворяющего условию (12), может служить $h(x)=x /\left(x^{2}+1\right)^{2}$, для которого $\widehat{h}_{s}(x)=\sqrt{\pi / 8} \cdot x \cdot \exp (-x)$. 
3. Теоремы существования и единственности. Используя теоремы 1 и 2 , можно доказать теоремы существования и единственности решения для различных классов нелинейных интегродифференциальных уравнений типа свертки. Для этого нам понадобится следующая теорема Ф. Браудера, приведенная с доказательством в [3, с. 98].

Теорема 3. Пусть $X-$ рефлексивное банахово пространство, $\Lambda \in\left(D(\Lambda) \rightarrow X^{*}\right)-$ радиально непрерывный максимальный монотонный оператор с линейной областью определения $D(\Lambda) \subset X$ и $A \in\left(X \rightarrow X^{*}\right)$ - радиально непрерывный монотонный коэриитивный оператор. Тогда при любом $f \in X^{*}$ уравнение $\Lambda u+A u=f$ имеет решение $u \in D(\Lambda)$. Если, кроме того, оператор $A$ является строго монотонным, то это решение единственно.

Заметим (см. [3, с. 143]), что теорема 3 была сформулирована Ф. Браудером без предположений радиальной непрерывности оператора $\Lambda$ и линейности области его определения $D(\Lambda)$.

Введем в рассмотрение нелинейный оператор суперпозиции (так называемый оператор Немыцкого). Пусть вещественнозначная функция $F(x, u)$ определена при $x \in[-\pi, \pi], u \in \mathbb{R}$, имеет период $2 \pi$ по $x$ и удовлетворяет условиям Каратеодори: она измерима по $x$ при каждом фиксированном $u \in \mathbb{R}$ и непрерывна по $u$ почти для всех $x \in[-\pi, \pi]$. Обозначим через $F$ оператор суперпозиции, порожденный функцией $F(x, u)$, а через $L_{p}^{+}(-\pi, \pi)$ - множество всех неотрицательных функций из $L_{p}(-\pi, \pi)$ с обычной нормой

$$
\|u\|_{p}=\left(\int_{-\pi}^{\pi}|u(x)|^{p} d x\right)^{1 / p}, \quad 1 \leqslant p<\infty .
$$

Рассмотрим сначала нелинейное интегро-дифференциальное уравнение типа свертки, наиболее простое для исследования методом максимальных монотонных операторов.

Теорема 4. Пусть $1<p<\infty, f(x) \in L_{p^{\prime}}(-\pi, \pi), h(x) \in L_{1}(-\pi, \pi)$ и удовлетворяет условию (4). Предположим, что для почти всех $x \in[-\pi, \pi]$ и всех $u \in \mathbb{R}$ выполняются следующие условия:

I.1. $|F(x, u)| \leqslant a(x)+d_{1} \cdot|u|^{p-1}$, где $a(x) \in L_{p^{\prime}}^{+}(-\pi, \pi), d_{1}>0$;

I.2. $F(x, u)$ не убывает по $u$;

I.3. $F(x, u) \cdot u \geqslant d_{2} \cdot|u|^{p}-D(x)$, где $D(x) \in L_{1}^{+}(-\pi, \pi), d_{2}>0$.

Тогда при любых значениях параметра $\lambda>0$ уравнение

$$
\lambda \cdot F(x, u(x))+\int_{-\pi}^{\pi} h(x-t) \cdot u^{\prime}(s) d s=f(x)
$$

имеет решение $и(x) \in M_{p}(-\pi, \pi)$. Это решение единственно, если в условии I.2 функция $F(x, u)$ строго возрастает по $u$.

Доказательство. Запишем уравнение (22) в операторном виде:

$$
\lambda \cdot F u+T u=f .
$$

Из условий I.1-І.3 вытекает, соответственно, что оператор Немыцкого $F$, порожденный функцией $F(x, u)$, действует непрерывно из $L_{p}(-\pi, \pi)$ в $L_{p^{\prime}}(-\pi, \pi)$, монотонен и коэрцитивен, причем для всех $u(x) \in L_{p}(-\pi, \pi)$ выполняются неравенства

$$
\|F u\|_{p^{\prime}} \leqslant\|a\|_{p^{\prime}}+d_{1} \cdot\|u\|_{p}^{p-1}, \quad\langle F u, u\rangle \geqslant d_{2} \cdot\|u\|_{p}^{p}-\|D\|_{1} .
$$

Из первого неравенства также вытекает, что нелинейный оператор $F$ является ограниченным.

Рассмотрим теперь интегро-дифференциальный оператор свертки $T$. При доказательстве теоремы 1 было установлено, что оператор $T$ действует из $D(T)=M_{p}(-\pi, \pi)$ в $L_{p^{\prime}}(-\pi, \pi)$ и является положительным, а значит, в силу линейности, и монотонным оператором. Кроме того, оператор $T$ является максимальным монотонным оператором, так как не имеет строгого монотонного расширения. Таким образом, для операторов $T=\Lambda$ и $\lambda \cdot F=A$ выполняются все требования теоремы 3 . 
Следовательно, уравнение (23), а значит, и уравнение $(22)$, имеет решение $u \in M_{p}(-\pi, \pi)$. Это решение единственно, если $F(x, u)$ в условии I.2 строго возрастает по $u$, поскольку в этом случае оператор $A=\lambda \cdot F$ будет строго монотонным.

Поскольку функция $F(x, u)=u^{p-1}(x)$, где $p \geqslant 2$ есть любое четное число, удовлетворяет условиям I.1-I.3 при $d_{1}=d_{2}=1$ и $a(x)=D(x)=0$, то справедливо следующее утверждение.

Следствие 1. Пусть $p \geqslant 2$ - любое четное число, $f(x) \in L_{p^{\prime}}(-\pi, \pi)$, а ядро $h(x) \in L_{1}(-\pi, \pi)$ удовлетворяет условию (4). Тогда уравнение

$$
u^{p-1}(x)+\int_{-\pi}^{\pi} h(x-t) \cdot u^{\prime}(t) d t=f(x)
$$

имеет единственное решение $u(x) \in M_{p}(-\pi, \pi)$.

Следующая теорема отличается от теоремы 4 как по характеру ограничений на нелинейность $F(x, u)$ и правую часть $f(x)$, так и по структуре доказательства, основанном на обращении нелинейного оператора суперпозиции и установлении коэрцитивности обратного оператора.

Теорема 5. Пусть $1<p<\infty, f(x) \in M_{p}(-\pi, \pi)$, а ядро $h(x) \in L_{1}(-\pi, \pi)$ удовлетворлет условию (4). Предположим, что для почти всех $x \in[-\pi, \pi]$ и всех $u \in \mathbb{R}$ выполняются следующие условия:

II.1. $|F(x, u)| \leqslant g(x)+d_{3} \cdot|u|^{1 /(p-1)}$, где $g(x) \in L_{p}^{+}(-\pi, \pi), d_{3}>0$;

II.2. $F(x, u)$ строго возрастает по u;

II.3. $F(x, u) \cdot u \geqslant d_{4} \cdot|u|^{p /(p-1)}-D(x)$, где $D(x) \in L_{1}^{+}(-\pi, \pi), d_{4}>0$,

то при любых значениях параметра $\lambda \geqslant 0$ уравнение

$$
u(x)+\lambda \cdot F\left(x, \int_{-\pi}^{\pi} h(x-t) \cdot u^{\prime}(t) d t\right)=f(x)
$$

имеет единственное решение $u(x) \in M_{p}(-\pi, \pi)$.

Доказательство. При $\lambda=0$ утверждение данной теоремы очевидно, поэтому считаем далее, что $\lambda>0$. Запишем уравнение $(24)$ в операторном виде:

$$
u+\lambda \cdot F T u=f
$$

и покажем, что оно имеет решение $u(x) \in M_{p}(-\pi, \pi)$. Для этого введем новую неизвестную функцию $v(x)$, обозначив $f(x)-u(x)=\lambda \cdot v(x)$. Ясно, что $v(x) \in M_{p}(-\pi, \pi)$. Подставив $u=f-\lambda \cdot v$ в уравнение $(25)$, получим:

$$
F T(f-\lambda \cdot v)=v .
$$

Из условий II.1-II.3 вытекает, что оператор Немыцкого $F$ действует непрерывно из сопряженного $L_{p^{\prime}}(-\pi, \pi)$ в исходное пространство $L_{p}(-\pi, \pi)$, строго монотонен и коэрцитивен, причем для любого $w(x) \in L_{p^{\prime}}(-\pi, \pi)$ выполняются неравенства

$$
\|F w\|_{p} \leqslant\|g\|_{p}+d_{3}\|w\|_{p^{\prime}}^{1 /(p-1)}, \quad\langle F w, w\rangle \geqslant d_{4}\|w\|_{p^{\prime}}^{p /(p-1)}-\|D\|_{1} .
$$

Значит, по [3, теорема 2.2]) существует обратный оператор $F^{-1}$, действующий из $L_{p}(-\pi, \pi)$ в $L_{p^{\prime}}(-\pi, \pi)$ и являющийся строго монотонным, ограниченным и радиально непрерывным (заметим, что для монотонных операторов понятия радиально непрерывный и деминепрерывный совпадают, в силу [3, лемма 1.3].

Покажем, что обратный оператор $F^{-1}$ является коэрцитивным, т.е. удовлетворяет условию

$$
\lim _{\|v\|_{p} \rightarrow \infty} \frac{\left\langle F^{-1} v, v\right\rangle}{\|v\|_{p}}=\infty .
$$

Пусть $v(x) \in L_{p}(-\pi, \pi)$ и $F^{-1} v=w$. Тогда в силу первого неравенства из (27) имеем

$$
\|v\|_{p}=\|F w\|_{p} \leqslant\|g\|_{p}+d_{3}\|w\|_{p^{\prime}}^{1 /(p-1)} .
$$


Следовательно, $\|w\|_{p^{\prime}} \rightarrow \infty$, если $\|v\|_{p} \rightarrow \infty$. Поэтому, используя неравенство (29) и второе неравенство из (27), получаем

$$
\frac{\left\langle F^{-1} v, v\right\rangle}{\|v\|_{p}}=\frac{\langle w, F w\rangle}{\|v\|_{p}} \geqslant \frac{d_{4}\|w\|_{p^{\prime}}^{p /(p-1)}-\|D\|_{1}}{\|g\|_{p}+d_{3}\|w\|_{p^{\prime}}^{1 /(p-1)}} \rightarrow \infty \quad x \text { при } \quad\|v\|_{p} \rightarrow \infty
$$

т.е. справедливо равенство (28), и поэтому оператор $F^{-1}$ является коэрцитивным.

Применив теперь оператор $F^{-1}$ к обеим частям уравнения $(26)$, имеем $T f-\lambda \cdot T v=F^{-1} v$, или

$$
F^{-1} v+\lambda \cdot T v=T f
$$

т.е. приходим к уравнению вида (23).

Поскольку операторы $\lambda \cdot T=\Lambda$ и $F^{-1}=A$ удовлетворяют всем требованиям теоремы 3 , то уравнение (31) имеет решение $v \in M_{p}(-\pi, \pi)$. Единственность этого решения вытекает из строгой монотонности оператора $F^{-1}$. Но тогда в силу связи $u=f-\lambda \cdot v$ уравнение $(25)$, а значит и данное уравнение (24), имеет единственное решение $u \in M_{p}(-\pi, \pi)$.

Следствие 2. Пусть $p \geqslant 2-$ любое четное число, $f(x) \in M_{p}(-\pi, \pi)$, а ядро $h(x) \in L_{1}(-\pi, \pi)$ удовлетворяет условию (4). Тогда уравнение

$$
u(x)+\left(\int_{-\pi}^{\pi} h(x-t) \cdot u^{\prime}(t) d t\right)^{1 /(p-1)}=f(x)
$$

имеет единственное решение $u(x) \in M_{p}(-\pi, \pi)$.

Из следствий 1 и 2 непосредственно вытекает, что теоремы 4 и 5 при $p=2$ охватывают, в частности, и случай линейных интегро-дифференциальных уравнений типа свертки.

Используя теорему 2 вместо теоремы 1 , аналогично теоремам 4 и 5 можно доказать теоремы о существовании и единственности решения в классе $M_{p}(\mathbb{R})$ для нелинейных интегро-дифференциальных уравнений типа свертки

$$
\lambda \cdot F(x, u(x))+\int_{-\infty}^{\infty} h(x-t) \cdot u^{\prime}(s) d s=f(x), \quad u(x)+\lambda \cdot F\left(x, \int_{-\infty}^{\infty} h(x-t) \cdot u^{\prime}(t) d t\right)=f(x)
$$

на всей действительной оси $\mathbb{R}$.

В заключение отметим, что методом максимальных монотонных операторов могут быть также изучены нелинейные сингулярные интегро-дифференциальные уравнения с ядрами Гильберта и Коши (см. [2]).

\section{СПИСОК ЛИТЕРАТУРЫ}

1. Асхабов C. Н. Нелинейные уравнения типа свертки. - М.: Физматлит, 2009.

2. Асхабов $C$. Н. Нелинейные сингулярные интегро-дифференциальные уравнения с произвольным параметром// Мат. заметки. - 2018. - 103, № 1. - С. 20-26.

3. Гаевский X., Грегер K., Захариас K. Нелинейные операторные уравнения и операторные дифференциальные уравнения. - М.: Мир, 1978.

4. Гахов Ф. Д., Черский Ю. И. Уравнения типа свертки. - М.: Наука, 1978.

5. Зигмунд А. Тригонометрические ряды. Т. 1. - М.: Мир, 1965.

6. Князев П. Н. Интегральные преобразования. - М.: Едиториал УРСС, 2004.

7. Харди Г. Х., Рогозинский В. В. Ряды Фурье. - М.: Физматгиз, 1959.

8. Хачатрян $X$. A. О разрешимости в $W_{1}^{1}(\mathbb{R})$ одного нелинейного интегро-дифференциального уравнения с некомпактным оператором Гаммерштейна-Немыцкого// Алгебра и анализ. -2012 . -24 , № 1 . C. $223-247$.

9. Эдвардс Р. Ряды Фурье в современном изложении. Т. 1. - М.: Мир, 1985.

10. Brunner H. Volterra Integral Equations: An Itroduction to Theory and Applications. - Cambridge: Cambridge Univ. Press, 2017. 
11. Gripenberg G., Londen S. O., Staffans O. Volterra Integral and Functional Equations. - Cambridge-New York: Cambridge Univ. Press, 1990.

Асхабов Султан Нажмудинович

Чеченский государственный педагогический университет, Грозный;

Чеченский государственный университет, Грозный

E-mail: askhabov@yandex.ru 